
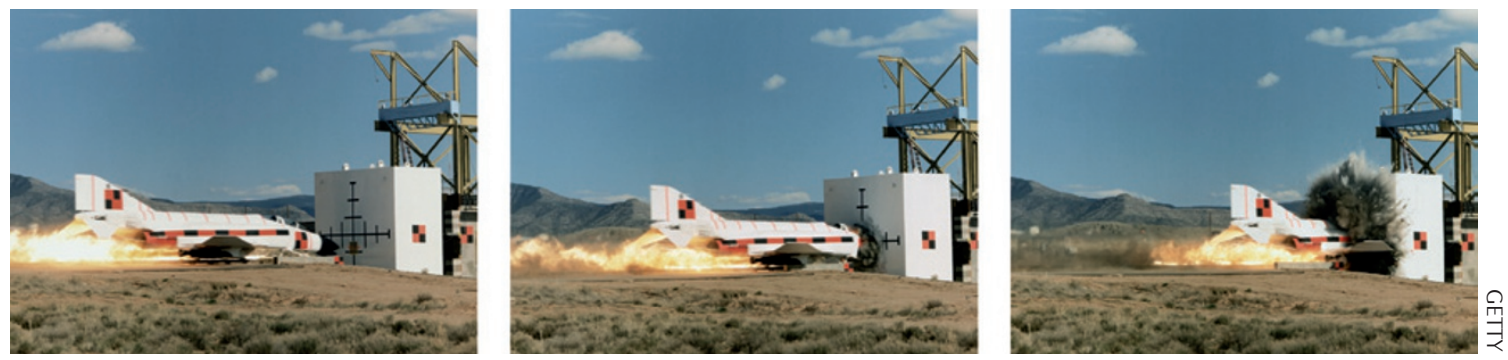

ANTIVIRAL IMMUNITY

\title{
SAMHD1 - stopping HIV in its tracks
}

HIV-1 replicates poorly in human dendritic cells (DCs), monocytes and, to a lesser extent, macrophages, but the restriction factor that mediates this non-permissiveness to infection remains unknown. Writing in Nature, Laguette et al. identify SAMHD1 (SAM- and HD-domaincontaining protein 1) as an HIV-1 restriction factor that is expressed by myeloid cells and blocks an early step in the life cycle of HIV-1.

Previous studies have shown that transduction of myeloid cells by virus-like particles containing viral protein $\mathrm{X}(\mathrm{Vpx})$, which is expressed by HIV-2 and simian immunodeficiency virus (SIV) but not by HIV-1, can counteract an antiviral restriction factor. This allows infection by these viruses, but also by other retroviruses, including HIV-1. Using tandem affinity chromatography purification coupled with mass spectrometry, and other approaches, the authors identified SAMHD1 as a Vpx-interacting protein in differentiated THP1 monocytic cells (which are non-permissive to HIV-1 infection). Exposure of THP1 cells to Vpx contained in virus-like particles resulted in reduced levels of SAMHD1, and this reduction was reversed by treatment with a proteasome inhibitor. These data suggest that Vpx induces proteasomal degradation of SAMHD1.

So, does SAMHD1 render myeloid cell types refractory to HIV-1 infection? SAMHD1 was found to be highly expressed by monocytes and monocyte-derived DCs, to be expressed at low levels by monocyte-derived macrophages, and to be absent from HIV-1-sensitive $\mathrm{T}$ cell lines. These expression profiles inversely correlate with the permissiveness of the cell types to HIV-1 infection. Knockdown of SAMHD1 expression in activated THP1 cells using short hairpin RNA resulted in increased permissiveness of these cells to HIV-1 infection, whereas expression of SAMHD1 in infectionpermissive differentiated U937 cells rendered them almost refractory to HIV-1 infection. Furthermore, silencing of SAMHD1 expression in primary human DCs increased their susceptibility to HIV-1 infection. Finally, SAMHD1 was found to exert its restrictive effects at the reverse transcription step of HIV-1 replication.

So, the expression of SAMHD1 by cells of the myeloid lineage renders them refractory to HIV-1 infection and these findings may be useful in the development of DC-targeted vaccines against HIV/AIDS.

Olive Leavy

ORIGINAL RESEARCH PAPER Laguette, N. et al. SAMHD1 is the dendritic- and myeloid-cell-specific HIV-1 restriction factor counteracted by Vpx. Nature 25 May 2011 (doi:10.1038/nature10117) 